\title{
A NOTE ON MULTIPLICATIVELY PRIME IDEALS OF OPERATORS
}

\author{
PEI-KEE LIN \\ (Communicated by William J. Davis)
}

\begin{abstract}
An ideal $\mathscr{I}$ of $\mathscr{L}(\mathscr{H})$ is said to be multiplicatively prime if $A X B \in \mathscr{I}$ for all $X \in \mathscr{L}(\mathscr{H})$ implies $A$ or $B$ is in $\mathscr{I}$. In this paper, we show there exists a nonnorm multiplicatively prime ideal other than $\mathscr{F}$.
\end{abstract}

An ideal $\mathscr{I}$ of $\mathscr{L}(\mathscr{H})$ is said to be square (respectively, multiplicatively prime) if $\mathscr{I}=\mathscr{I}^{2}$ (respectively, $A X B \in \mathscr{I}$ for all $X \in \mathscr{L}(\mathscr{H}$ ) implies $A$ or $B$ in $\mathscr{I}$ ). An ideal $\mathscr{I}$ is said to have the square root property if $\sqrt{|A|} \in \mathscr{I}$ for all $A \in \mathscr{I}$. R. I. Loebl [L] proved that if $\mathscr{I}$ is multiplicatively prime then $\mathscr{I}$ is square and $\mathscr{I}$ has the square root property. He also showed that these properties are equivalent if $\mathscr{I}$ is a proper norm ideal. (In this article, norm ideals are named "symmetrically normed Banach ideals" [G-K, p. 68]. A symmetrically normed ideal is said to be a nonnorm ideal if it is not a Banach space under any symmetrical norm.) In this case, $\mathscr{I}$ is either $\{0\}$ or $\mathscr{K}$, the ideal of all compact operators. He asked whether there exists a nonnorm multiplicatively prime ideal other than $\mathscr{F}$, the ideal of all finite rank operators. The purpose of this paper is to show there exists such an ideal.

Let $\left\{a_{i}\right\},\left\{b_{i}\right\}$ be two decreasing sequences whose limits are zero. Then $\left\{a_{i}\right\} \oplus$ $\left\{b_{i}\right\}=\left\{c_{i}\right\}$ denotes the decreasing sequence which is the union of $\left\{a_{i}\right\}$ and $\left\{b_{i}\right\}$ (counting multiplicity). For any natural number $n, n \odot\left\{a_{i}\right\}$ denotes the sequence

$$
\left\{a_{i}\right\} \oplus\left\{a_{i}\right\} \oplus^{n \text { times }} \oplus\left\{a_{i}\right\} .
$$

Recall that an ideal set $I$ is a collection of decreasing sequences $\left\{a_{i}\right\}$ with the following properties:

(i) If $\left\{a_{n}\right\} \in I$, then $\lim _{n \rightarrow \infty} a_{n}=0$.

(ii) If $\left\{a_{n}\right\} \in I$ and $\left\{b_{n}\right\}$ is a decreasing sequence such that $0 \leqslant b_{n} \leqslant a_{n}$ for all $n$, then $\left\{b_{n}\right\} \in I$.

(iii) If $\left\{a_{n}\right\} \in I$ and $c>0$ then $\left\{c a_{n}\right\} \in I$.

(iv) If $\left\{a_{n}\right\},\left\{b_{n}\right\} \in I$, then $\left\{a_{n}\right\} \oplus\left\{b_{n}\right\} \in I$.

An ideal set $I$ is said to have the square root property if $\left\{a_{n}\right\} \in I$ implies $\left\{a_{n}^{1 / 2}\right\} \in I$. An ideal set $I$ is said to be multiplicatively prime if for any two decreasing sequences $\left\{a_{n}\right\},\left\{b_{n}\right\},\left\{a_{n} b_{n}\right\} \in I$ implies $\left\{a_{n}\right\} \in I$ or $\left\{b_{n}\right\} \in I$. It is

Received by the editors April 10, 1986 and, in revised form, August 10, 1986.

1980 Mathematics Subject Classification (1985 Revision). Primary 47D25.

Supported in part by NSF Grant DMS-8514497.

(C)1987 American Mathematical Society $0002-9939 / 87 \$ 1.00+\$ .25$ per page 
known [ $\mathbf{C}$ and $\mathbf{F}-\mathbf{L}$ ] that there is a bijection between (multiplicatively prime) proper two-sided ideals and (multiplicatively prime) ideal sets. Hence, we only need to construct a nontrivial multiplicatively prime ideal set. We need a couple of lemmas.

LEMMA 1. Suppose the ideal set $I$ has the square root property and $\left\{a_{n}\right\}$ is a decreasing sequence which is not in I. Let $\left\{b_{n}\right\}$ and $\left\{c_{n}\right\}$ be two decreasing sequences such that $\left\{b_{n} c_{n}\right\} \in I$. Then there exists an ideal set $I^{\prime} \supseteq I$ with the square root property so that either $\left\{b_{n}\right\}$ or $\left\{c_{n}\right\}$ is in $I^{\prime}$ but $I^{\prime}$ does not contain $\left\{a_{n}\right\}$.

LEMma 2. Suppose the ideal set I has the square root property and $\left\{a_{n}\right\}$ is a decreasing sequence which is not in I. Then there exists a multiplicatively prime ideal set $I^{\prime} \supseteq I$ such that $\left\{a_{n}\right\} \notin I^{\prime}$.

Proof of Lemma 2. Suppose Lemma 1 were proved. Let $\mathscr{D}$ be the collection of the ideal set $I^{\prime} \supseteq I$ with the square root property such that $\left\{a_{n}\right\} \notin I^{\prime}$. If $I^{\prime}$ and $I^{\prime \prime}$ are in $\mathscr{D}$, we say $I^{\prime} \leqslant I^{\prime \prime}$ whenever $I^{\prime} \subseteq I^{\prime \prime}$. Then $(\mathscr{D}, \leqslant)$ satisfies the assumption of Zorn's Lemma, and $\mathscr{D}$ contains a maximal element $I^{\prime}$. By Lemma $1, I^{\prime}$ is multiplicatively prime.

By Lemma 2 and Theorem 3 in [L], we have the following theorem.

THEOREM. $\mathscr{F}$ is the unique nonnorm multiplicatively prime ideal if (and only if) $\mathscr{F}$ is the unique nonnorm ideal with the square root property.

REMARK 1. Since

$$
\mathscr{I}_{p}=\bigcup_{k \geqslant 0} C_{p 2^{k}} \neq \mathscr{F}
$$

is a nonnorm ideal with the square root property $[\mathbf{L}]$, there exists a nonnorm multiplicatively prime ideal other than $\mathscr{F}$.

REMARK 2. Let $\left\{a_{n}\right\}$ and $\left\{b_{n}\right\}$ be two sequences such that $\lim _{n \rightarrow \infty} a_{n}=0=$ $\lim _{n \rightarrow \infty} b_{n}$. We say $\left\{a_{n}\right\} \leqslant\left\{b_{n}\right\}$ if there exists $N$ such that $a_{n} \leqslant b_{n}$ whenever $n>N$. If $c \geqslant c^{\prime} \geqslant 0, m \geqslant m^{\prime} \geqslant 1, k \geqslant k^{\prime} \geqslant 0$, and $\left\{a_{n}\right\} \leqslant\left\{b_{n}\right\}$, then

$$
m^{\prime} \odot\left\{c^{\prime} a_{n}^{1 / 2^{h \prime}}\right\} \leqslant m^{\prime} \odot\left\{c^{\prime} a_{n}^{1 / 2^{h}}\right\} \leqslant m \odot\left\{c a_{n}^{1 / 2^{k}}\right\} \leqslant m \odot\left\{c b_{n}^{1 / 2^{k}}\right\} .
$$

If $I$ is an ideal set, $\left\{b_{n}\right\} \in I$, and $\left\{a_{n}\right\} \leqslant\left\{b_{n}\right\}$, then by (ii) and (iii) $\left\{a_{n}\right\} \in I$.

REMARK 3. Suppose $I$ is an ideal set with the square root property. Let $\left\{a_{n}\right\}$ be a decreasing sequence such that $\lim _{n \rightarrow \infty} a_{n}=0$. Then the smallest ideal set with the square root property which contains $I$ and $\left\{a_{n}\right\}$ consists of all sequences $\left\{b_{n}\right\}$ with the following property: There exist $c \geqslant 0, m \geqslant 1, k \geqslant 0$, and $\left\{c_{n}\right\} \in I$ such that

$$
\left\{b_{n}\right\} \leqslant m \odot\left\{c a_{n}^{1 / 2^{k}}\right\} \oplus\left\{c_{n}\right\} .
$$

Proof of Lemma 1. Suppose Lemma 1 is not true. Then there exist an ideal set $I$ with the square root property and three decreasing sequences $\left\{a_{n}\right\},\left\{b_{n}\right\},\left\{c_{n}\right\}$ such that $\left\{a_{n}\right\} \notin I,\left\{b_{n}\right\} \notin I,\left\{c_{n}\right\} \notin I, \quad\left\{a_{n} b_{n}\right\} \in I$, and the smallest ideal set $I_{1}$ (respectively $I_{2}$ ) which contains $I$ and $\left\{a_{n}\right\}$ (respectively $\left\{b_{n}\right\}$ ) contains $\left\{c_{n}\right\}$. For fixed $c>0, m>0$, and $k \geqslant 0$, if $\left\{d_{n}\right\}=m \odot\left\{c a_{n}^{1 / 2^{k}}\right\}$ and $\left\{d_{n}^{\prime}\right\}=m \odot\left\{c b_{n}^{1 / 2^{h}}\right\}$ then $\left\{d_{n} d_{n}^{\prime}\right\} \in I$. By Remarks 2 and 3 , we may assume that there exist $\left\{e_{n}\right\}$, 
$\left\{e_{n}^{\prime}\right\} \in I$ such that

$$
\left\{c_{n}\right\} \leqslant\left\{a_{n}\right\} \oplus\left\{e_{n}\right\}=\left\{f_{n}\right\}
$$

(respectively, $\left\{c_{n}\right\} \leqslant\left\{b_{n}\right\} \oplus\left\{e_{n}^{\prime}\right\}=\left\{f_{n}^{\prime}\right\}$ ). One can verify that $\left\{f_{n} f_{n}^{\prime}\right\} \leqslant\left\{a_{n} b_{n}\right\} \oplus$ $\left\{e_{n}\right\} \oplus\left\{e_{n}^{\prime}\right\} \in I$. This implies $\left\{c_{n}^{2}\right\} \in I$. But $I$ has the square root property. So $\left\{c_{n}\right\} \in I$ and we get a contradiction.

\section{REFERENCES}

[C] J. W. Calkin, Two-sided ideals and convergences in the ring of bounded operators in Hilbert space, Ann. of Math. (2) 42 (1941), 837-873.

[F-L] L. Fialkow and R. Loebl, Elementary mappings into ideals of operators, Illinois J. Math. 28 (1984), 555-578.

[K-G] I. C. Gohberg and M. G. Krein, Introduction to the theory of linear nonselfadjoint operators, Transl. Math. Monographs, vol. 18, Amer. Math. Soc., Providence, R.I., 1969.

[L] R. I. Loebl, A note on ideals of operators, Proc. Amer. Math. Soc. 96 (1986), 62-64.

Department of Mathematics, University of Iowa, Iowa City, Iowa 52242

Current address: Department of Mathematics, Memphis State University, Memphis, Tennessee 38152 